\title{
Data Analytics through BlockChain Technologies
}

\author{
Ch.Sekhar ${ }^{1}$ and N Tirupathi Rao $^{2}$ \\ Dept of Comp. Science and Engineering, Vignan's Institute of IT (A), Visakhapatnam, \\ AP, India \\ ${ }^{1}$ sekhar1203@gmail.com, ${ }^{2}$ nakkathiru@gmail.com
}

\begin{abstract}
Huge Information is a term for a considerable amount, not predictable, emergent data collections among abundant, self-reliant sources. Created in different fields extending from monetary and business exercises to open organization, plays a significant role to take any decision. Blockchain is a dispersed database framework that goes about as an "open record" to store and oversee exchanges. Each trace in the DB(Database) is known as a square and contain points of interest, for example, the exchange timestamp and besides a connection to the past square. Makes it unthinkable for everybody to change data about the records thoughtfully. Furthermore, since the way that a similar exchange can record over the various, dispersed database structure, the innovation is secure by the plan. This paper provides the fundamentals of big data and how we can use the blockchain technology.
\end{abstract}

Keywords: Data, Analytics, Block Chain, Decentralization

\section{Introduction}

In the path of current decades, information has stretched on a massive scale in different fields. As per details from International Data Corporation (IDC), in 2011, the general made and replicated information volume on the planet was $1.8 \mathrm{ZB}$, which expanded by about nine times inside five years [1]. This figure will twofold somewhere around each other two years sooner rather than later. Under the hazardous development of data, the term Big Data implies the vast or complex informational indexes that couldn't be dealt with by customary database advances. Massive Data likewise emerges with numerous difficulties, for example, troubles in information catch, information stockpiling, information investigation and information representation. Enormous information is described by the five Vs, to be specific volume, assortment, speed, esteem and veracity.

Blockchain, the foundation of BITCOIN, has gotten across the board considerations lately. Blockchain fills in as an unchallengeable record which permits the business to happen in a decentralized methodology. Blockchain-based applications are jumping up and spread different fields to incorporate monetary administrations, notoriety framework and IoT. In any case, there are as yet numerous difficulties of blockchain innovation, for example, versatility and security issues holding back to be survived. This paper presents a comprehensive outline of blockchain innovation. We clarified a diagram of blockchain design right off the bat and looked at several average properties utilized in various blockchains. Moreover, specialized

Article history:

Received (January 27, 2020), Review Result (March 5, 2020), Accepted (April 14, 2020) 
difficulties and late advances can quickly be recorded. We additionally spread out conceivable future patterns for blockchain [1].

\section{Data analytics}

Envision a world without information stockpiling; a place where everything about an individual or association, each exchange performed, or each angle which can be recorded is lost straightforwardly after use. Associations would, in this manner, lose the capacity to remove critical data and information, perform itemized examinations, just as give new chances and preferences. Anything extending from client names and addresses to items accessible, to buys made, to workers procured, and so on has gotten essential for everyday congruity. Information is the structure hinder whereupon any association thrives [2].

The articulation "Gigantic Data" has to start fresh associated with datasets that grow so significant that they end up awkward to work with using standard database organization structures. They are instructive records whose gauge is past the limit of usually used programming gadgets and limit systems to get, store, direct, and process the data inside a widely appealing took a break. Large data sizes are consistently growing, at present, stretching out from two or three dozen terabytes (TB) to various petabytes (PB) of data in a lone enlightening file. In this way, a part of the difficulties related to gigantic data fuse gets, amassing, look for, sharing, assessment, and envisioning. Today, endeavours are researching vast volumes of very organized data to discover facts they didn't know previously [3].

This $5 \mathrm{~V}$ definition features the importance and need of enormous information.

\subsection{Volume}

Volume implies, with the age and gathering of masses of information. These days volume or the extent of information in various zones/undertakings is more significant than terabytes and petabytes. For example, Twitter information channels, clickstreams on a website page and a versatile application arrange an activity, sensor-empowered gear catching information at the speed of light, and some more. Huge Data volume incorporates such highlights as size, scale, sum, the measurement for tera-and exascale information gathered from numerous exchanges and put away in individual records or databases to be available, accessible, handled and reasonable.

\subsection{Variety}

Variety manages the multifaceted nature of enormous information and data and semantic models behind this information. Vast information originates from an incredible assortment of sources and for the most part, has in four kinds: organized, semi-organized, unstructured and blended information. Organized information embeds an information distribution centre effectively labelled and effortlessly arranged; however, unstructured information is arbitrary and hard to examine. Unstructured and semi-organized information types, for example, content, sound, and the video does not comply with settled fields but preferably contains labels to isolate information components where they require extra handling to both infer meaning and the supporting metadata.

\subsection{Velocity}

Speed is the quick rate at which Big information streams is created into memory or plates by varieties of sensors or various occasions, and should be prepared progressively, close 
ongoing or in the cluster, or as streams (like in the event of perception). The speed required for vast information, as well as all procedures. For time-constrained procedures, enormous information ought to be utilized as it streams into the association with the end goal to augment its esteem [4]. A portion of the applications like Internet of Things (IoT), customer eCommerce and versatile communications . manage the large measure of information in their usage continuously or close ongoing.

\subsection{Value}

Assortment shows the different kinds of information, which include semi-organized and unstructured data, for example, sound, video, website page, and content, and in addition, current organized information. Esteem is a critical component of the Big information which is characterized by the additional esteem that the gathered information can convey to the planned procedure, movement or prescient examination/speculation. Information esteem will rely upon the occasions or procedures they speak to, for example, stochastic, probabilistic, standard or arbitrary. For instant in purchaser applications, the characteristic estimation of information is determined to utilize quantitative and insightful strategies from finding a customer inclination or conclusion to making a generous offer by area, or for recognizing a bit of gear that is going to come up short. Contingent upon this the prerequisites might be forced to gather all information, store for a more extended period (for some conceivable occasion of intrigue), and so forth. Nonetheless, discovering the estimation of Bigdata requires new revelation procedures to settle on more exact and exact choices.

\subsection{Veracity}

Enormous Data veracity guarantees that the information utilized are trusted, valid and shielded from unapproved access and change. The information must be anchored amid the entire their lifecycle from gathering from believed sources to preparing on confided in register offices and capacity on ensured and confided away offices. Information veracity depends altogether on the security framework conveyed and accessible from the Big Data foundation.

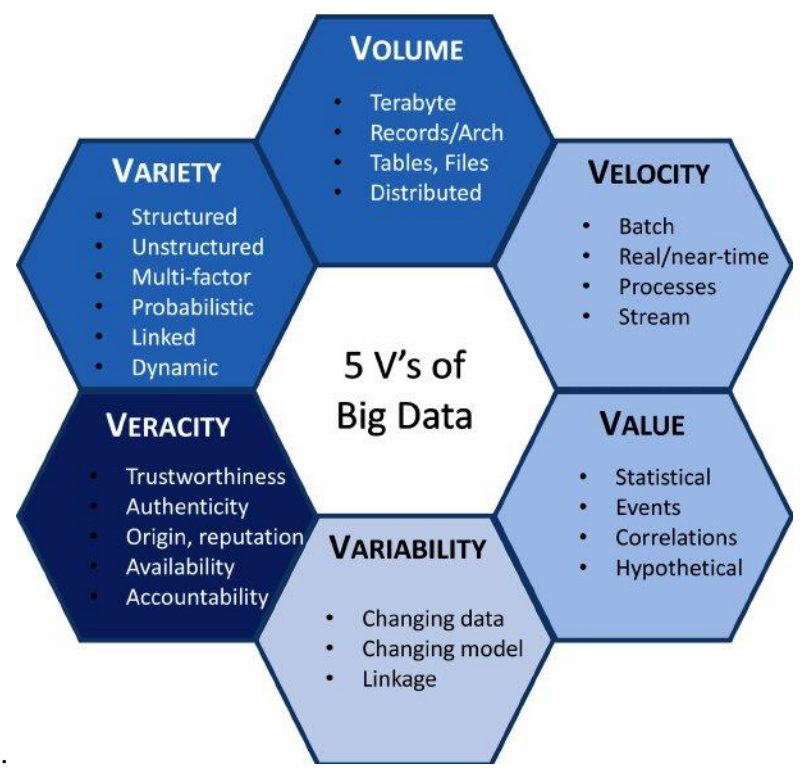

Figure 1. Properties of big data as 5 V's 
With this definition, qualities of huge information might be abridged as five Vs,i.e., Volume(significant volume), Variety (different modalities), Velocity (quick age), Value (gigantic esteem yet low thickness) and Veracity (trusted and accurate) as appeared in [Figure.1]

\section{BlockChain concept}

Blockchain is a database, or maybe more precisely, a record. Nonetheless, rather than information existing in sections and lines like in a regular database, it is contained in hinders that are fastened together in succession henceforth the name.

\subsection{Qualities}

Understanding blockchain requires attention to its particular qualities, which together take care of numerous issues of common information frameworks. Of these qualities, the accompanying five are especially prominent:

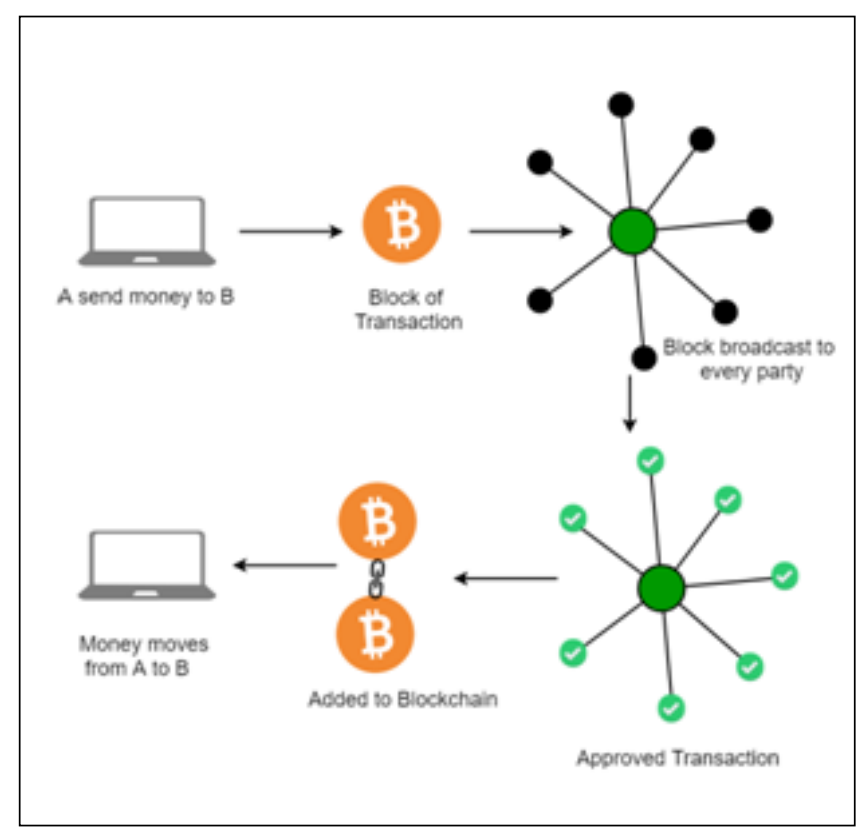

Figure 2. Illustration of BlockChain Process

- Blockchain records are decentralized: Unlike customary databases that can hold tight a single PC or server, blockchains can pass on records, genuinely open to any social affair in the framework. In a blockchain, there is no single motivation behind control.

- Peer-to-peer information transmission: As it is brought together purpose of control, blockchain information is transmitted legitimately starting with one client then onto the next. Every single transmitted datum in a flash updates the whole record, so everybody on the system has a similar single adaptation of reality.

- Anonymous exchanges: Data transmissions occur between addresses on the blockchain. This guarantees straightforwardness and, simultaneously, permits clients 
to stay unknown. Be that as it may, secrecy isn't essential if the blockchain is set up to require pieces of evidence of personality.

- Transaction records are changeless: Each blockchain exchange makes a record that is irreversible and unchangeable. Records are requested sequentially and are unmistakable to all clients in the chain.

- Blockchains are programmable: Because a blockchain can be bound to computational rationale, it's conceivable to program capacities and exchanges by means of rules and techniques.

\subsection{Major Components involved in Blockchain Architecture}

- Node- Node is the independent unit within the blockchain, i.e. user or computer

- Transaction - the minimum building block of a blockchain system (records, information, etc.) that serves as the purpose of blockchain

- Block - a data structure used for keeping a set of transactions which is distributed to all nodes in the network

- Chain: blocks are placed in a succession of blocks with detailed order

- Miners - detailed nodes which execute the block verification process before accumulation anything to the blockchain structure

- Consensus Protocol - a set of rules and preparations to carry out blockchain operations

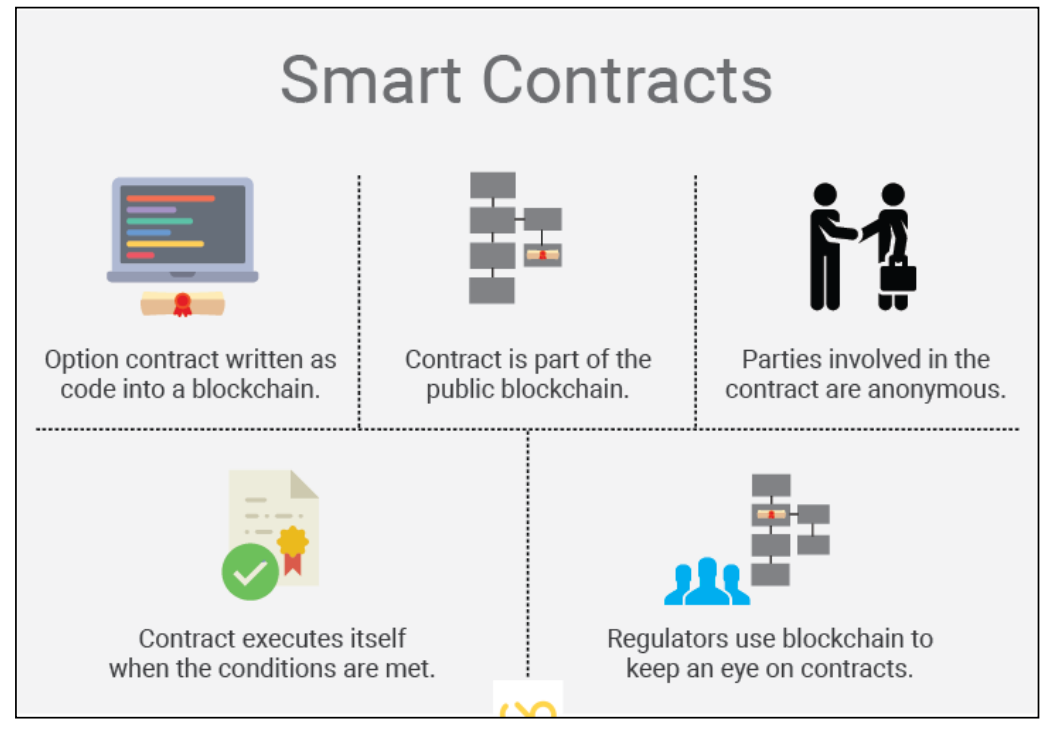

Figure 3. Financial application with blockchain technology

The above figure shows that smart contract application process with blockchain technology, initially contract between two organizations are placed on the blockchain as part of the public blockchain, and both parties are involved in the contract process anonymously. Once both parties agreed then the contract executes itself based on the constraints of the parties. Once the contract is finalized with the help of blockchain, regular monitoring can take place on the contract to avoid tempering.

\section{Data analytics with blockchain}


Utilizing square chain adds another information layer to the Big Data analytics process. In particular, this information layer follows 2 fundamental requests of the Big Data investigation:

Block chain-created Big Data is secure, as it can't be manufactured because of the system engineering. Block chain-based Big Data is significant, which means it is organized, plenteous and finish, making it an ideal hotspot for further examination. The information in the record can identify with vitality exchanging, land and an assortment of different areas. There are various Big Data investigation enhancements coming from this reality. For example, misrepresentation avoidance, as the blockchain innovation, permits the money related establishments to check each exchange continuously. Along these lines stated, rather than breaking down the records of the extortion that previously occurred, the banks can distinguish unsafe or deceitful exchanges on the fly and keep the misrepresentation totally [4][5].

\subsection{Opportunities for big data analytics}

As of late, a consortium of 47 Japanese banks joined with a blockchain startup called Ripple to encourage cash exchanges between ledgers utilizing blockchain. The principle explanation for the move is to perform continuous exchanges at a fundamentally minimal effort. One reason customary constant exchange was costly was a direct result of the potential hazard factors. Twofold spending (which is a type of exchange disappointment where a similar security token gets utilized twice) is a genuine issue with constant exchanges. With blockchains, that chance is, to a great extent, evaded. Enormous information investigation makes it conceivable to distinguish designs in purchaser spending and recognize dangerous exchanges much snappier than they should be possible as of now. This decreases the expense with continuous exchanges [6]

In Industries outside of managing an account as well, the fundamental drive for selection of Blockchain advancements has been security. Crosswise over medicinal services, retail and open organization, foundations have begun trying different things with blockchain to deal with information to forestall hacking and information spills. In social insurance and innovation, for example, blockchain can ensure that numerous "marks" are looked for at each level of information get to. This can help keep a rehash of occasions, for example, the 2015 assault that prompted the burglary of more than 100 million patient records.

\subsection{Potential outcomes in real-time analytics}

Up to this point, constant extortion discovery has just been a pipe dream and saving money foundations have dependably depended on utilizing advancements to recognize deceitful exchanges reflectively. Since the blockchain has a database record for each and every exchange, it gives an approach to establishments to dig for examples continuously, if required.

Be that as it may, these potential outcomes likewise bring up issues about protection, and this is in direct logical inconsistency to the motivation behind why blockchain and bitcoins wound up well known in any case. A few industry specialists have communicated worries that an innovation that can give a record of each exchange can be misused for everything "from client profiling to different less kind reasons".

From another point of view, in any case, blockchains enormously enhance straightforwardness in information examination. In contrast to past calculations, the blockchain configuration rejects any information that it can't check and is regarded as suspicious. Accordingly, experts in ventures, for example, Retail just manage information that is totally straightforward. As such, the client standards of conduct that blockchain frameworks distinguish are probably going to be a mess more exact than it is today. 


\subsection{Revealing transactional data}

The information inside the blockchain is anticipated to be worth trillions of dollars as it keeps on advancing into managing an account, micropayments, settlements, and other money related administrations. Truth be told, the blockchain record could be worth up to $20 \%$ of the aggregate huge information showcase by 2030 , creating up to $\$ 100$ billion in yearly income. To place this into point of view, this potential income outperforms that of what Visa, MasterCard, and PayPal at present produce combined. Big information investigation will be urgent in following these exercises and helping associations utilizing the blockchain settle on more educated choices [7]. Information insight administrations are developing to encourage monetary foundations, governments, and a wide range of associations dig into whom they may collaborate on the blockchain and reveal "concealed" designs.

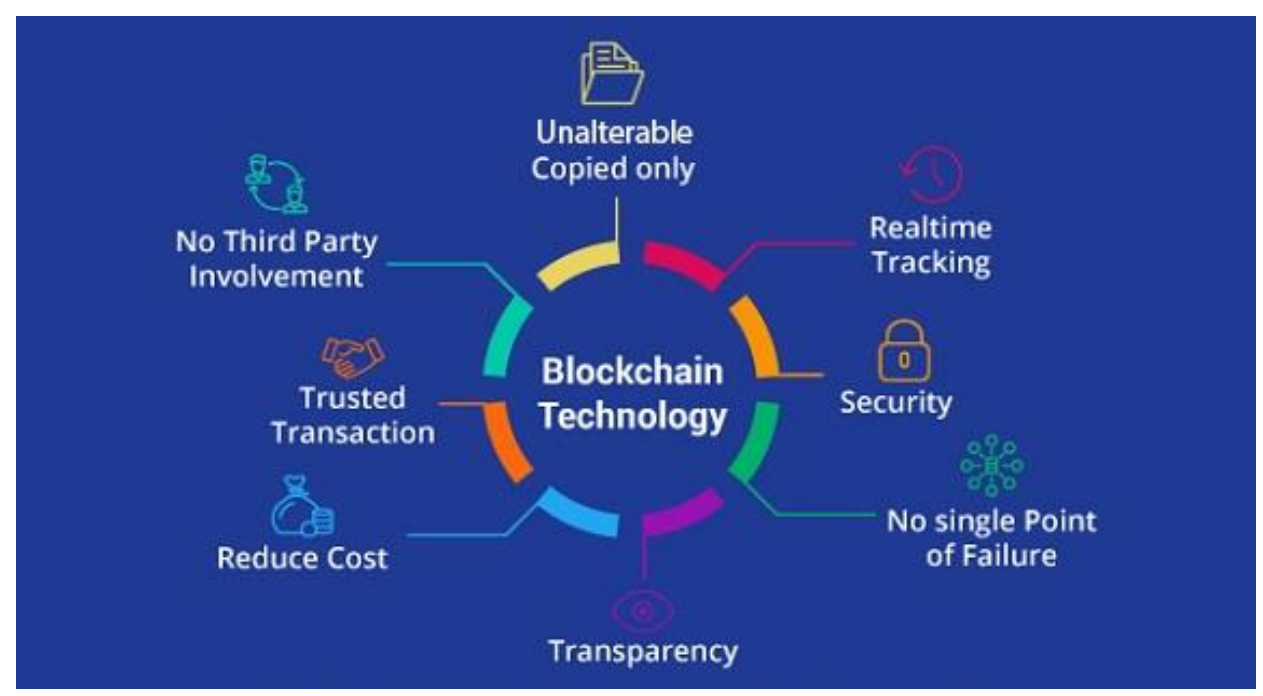

Figure 3. Benefits of blockchain

Applications of blockchain technologies are majorly in the financial sector like payment system, billing of lading, international currency exchange, public records, post-trade settlements retail banking and many more.

\section{Summary}

In the paper, we mention the need for data analytics with blockchain concept. The mix of blockchain and enormous information will help endeavours by making constant examination significantly more attainable and dependable. For instance, in the money related administrations industry, enormous information has not yet comprehended the troubles of recognizing misrepresentation and surveying hazard. This is fundamentally in light of the fact that current identification and evaluation techniques rely upon authentic information. In the event that money related establishments can saddle blockchain as a method for directing exchanges, at last, have the option to assess chance and distinguish suspicious examples progressively. This will assist with shielding banks and their clients from misrepresentationhowever, that is not all. It will likewise accelerate the exchange procedure (making it practically prompt) and lessen the expense of cash moves by disposing of the hindrances of security and hazard checks included. 


\section{References}

[1] Z. Zheng, S. Xie, H. Dai, X. Chen, and H. Wang, "An overview of blockchain technology: Architecture, consensus, and future trends," IEEE 6th International Congress on Big Data, (2017)

[2] Ch. Chandra Sekhar, J. Udaykumar, B. Kumar, and Ch. Sekhar, "Big Data analytics on Indian crop planning to increase agricultural production," Advanced Science and Technology Letters, (2017)

[3] Ch. Sekhar, N Suneetha, A. Sharma, and P. Sandhya, "Comprehensive analysis of hadoop ecosystem components: MapReduce, Pig and Hive,” Advanced Science and Technology Letters, vol.147, pp.468-478, (2017) DOI:10.14257/astl.2017.147.66

[4] S. Johansen, "A comprehensive literature review on blockchain technology as a technological enabler for innovation", (2016)

[5] Venkat, Introduction to blockchains \& what it means to big data, https://www.kdnuggets.com/2017/09/ introduction-blockchain-big-data.html, (2017)

[6] J. Moura and C. Serrão, "Security and privacy issues of big data," Handbook of Research on Trends and Future Directions in Big Data and Web Intelligence, (2015) DOI:10.4018/978-1-4666-8505-5.ch002

[7] D. Srinivasa Rao and V. Sucharita, "Analysis of different utility mining methodologies in transactional databases,” International Journal of Big Data Security Intelligence, vol.3, no.1, (2016) 